\title{
Uso da internet voltada ao público adolescente na atenção primária à saúde
}

\author{
Use of the internet aimed at the adolescent public in primary health care
}

Uso de internet dirigido al público adolescente en la atención primaria de salud

Mariana Ferreira Scope ${ }^{1 *}$, Graciela Dutra Sehnem ${ }^{1}$, Giovana Sangiogo Dallabrida ${ }^{1}$, Amanda Suélen Monteiro ${ }^{1}$, Anahy da Silva Machado ${ }^{1}$, Francielle Morais de Paula ${ }^{1}$, Silvana Bastos Cogo ${ }^{1}$, Eliane Tatsch Neves ${ }^{1}$.

\section{RESUMO}

Objetivo: Identificar as evidências do uso da internet como ferramenta de apoio voltada ao público adolescente na atenção primária à saúde. Métodos: Trata-se de um estudo de revisão integrativa da literatura, realizado via Portal PubMed e Biblioteca Virtual em Saúde (BVS). Foram utilizados no portal PubMed os seguintes descritores e operadores boleanos: [Internet] AND [Teenager] AND [Primary health care]. No portal BVS, utilizou-se os descritores [Internet] AND [Teenager] AND [Primary health care] OR [Internet] AND [Adolescente] AND [Atenção primária em saúde]. Incluíram-se artigos originais nos idiomas português, inglês ou espanhol, dos últimos cinco anos, com a intenção de identificar a produção de conhecimento atual acerca dessa temática. Resultados: $O$ corpus foi composto por nove estudos e a análise e síntese dos dados possibilitou agrupá-los em três categorias temáticas: Intervenções online que visam a prevenção de agravos e promoção da saúde dos adolescentes; Ferramentas adotadas pelo serviço para assistência à saúde mental dos adolescentes; e Uso da internet pelos adolescentes como fonte de informação em saúde. Considerações finais: Os meios tecnológicos podem melhorar significativamente a adesão dos adolescentes à procura dos serviços de saúde ou fornecer de forma virtual informação de qualidade, contribuindo também para o vínculo adolescente-profissional de saúde.

Palavras-chave: Internet, Adolescentes, Atenção primária à saúde, Enfermagem.

\begin{abstract}
Objective: To identify the evidence of the use of the internet as a support tool aimed at the adolescent public in primary health care. Methods: This is an integrative literature review study, carried out via the PubMed Portal and Virtual Health Library (VHL). The following Boolean descriptors and operators were used in the PubMed portal: [Internet] AND [Teenager] AND [Primary health care]. In the VHL portal, the descriptors [Internet] AND [Teenager] AND [Primary health care] OR [Internet] AND [Adolescent] AND [Primary health care] were used. Original articles in Portuguese, English or Spanish from the last five years were included, with the intention of identifying the production of current knowledge about this theme. Results: The corpus was composed of nine studies and the analysis and synthesis of the data made it possible to group them into three thematic categories: Online interventions aimed at preventing diseases and promoting the health of adolescents; Tools adopted by the service to assist adolescents' mental health; and Internet use by adolescents as a source of health information. Final considerations: Technological means can significantly improve the adherence of adolescents in search of health services or provide virtual information of quality, also contributing to the bond between adolescents and health professionals.
\end{abstract}

Key words: Internet, Adolescents, Primary health care, Nursing.

\footnotetext{
${ }^{1}$ Universidade Federal de Santa Maria (UFSM), Santa Maria - RS.

*E-mail: maryana.scopel@gmail.com
}

SUBMETIDO EM: 3/2021 


\section{RESUMEN}

Objetivo: Identificar la evidencia del uso de internet como herramienta de apoyo dirigida al público adolescente en la atención primaria de salud. Métodos: Se trata de un estudio de revisión integradora de la literatura, realizado a través del Portal PubMed y la Biblioteca Virtual en Salud (BVS). Los siguientes operadores y descriptores booleanos se utilizaron en el portal PubMed: [Internet] Y [Adolescente] Y [Atención primaria de salud]. En el portal de la BVS se utilizaron los descriptores [Internet] Y [Adolescente] Y [Atención primaria de salud] $O$ [Internet] $Y$ [Adolescente] $Y$ [Atención primaria de salud]. Se incluyeron artículos originales en portugués, inglés o español de los últimos cinco años, con la intención de identificar la producción de conocimiento actual sobre este tema. Resultados: El corpus estuvo compuesto por nueve estudios y el análisis y síntesis de los datos permitió agruparlos en tres categorías temáticas: Intervenciones en línea dirigidas a prevenir enfermedades y promover la salud de los adolescentes; Herramientas adoptadas por el servicio para ayudar a la salud mental de los adolescentes; y el uso de Internet por adolescentes como fuente de información sanitaria. Consideraciones finales: Los medios tecnológicos pueden mejorar significativamente la adherencia de los adolescentes a la búsqueda de servicios de salud o brindar información virtual de calidad, contribuyendo también al vínculo entre los adolescentes y los profesionales de la salud.

Palabras clave: Internet, Adolescentes, Atención primaria de salud, Enfermería.

\section{INTRODUÇÃO}

A adolescência consiste em uma das fases mais importantes da vida em relação ao desenvolvimento fisiológico, psicológico e social, sendo permeada por inúmeras peculiaridades, descobertas, mudanças, incertezas, escolhas e transformações (BRASIL, 2017). Esse período pode proporcionar momentos de instabilidade emocional tanto para o adolescente quanto para familiares e amigos que o cercam (COSTA RF, et al., 2015).

A Atenção Primária à Saúde (APS) precisa desempenhar um papel protagonista em relação a uma assistência integral desde a promoção à saúde até a prevenção e redução de danos para a população assistida, assegurando um acesso de qualidade aos serviços e políticas públicas com ações e diretrizes que atendam todas as necessidades (PINTO AC, et al., 2017). Entretanto, as ações voltadas para o público de adolescentes ainda apresentam fragilidades e inconsistências, o que reflete na ausência do adolescente nesse serviço, o que pode ser acentuado pela falta de vínculo com os profissionais. Tal realidade é resultado de uma carência no planejamento de ações nesses espaços, tendo em vista que a demanda espontânea não abrange, em sua totalidade, as particularidades deste público (PESSOA DMS, et al., 2020).

Assim, com o intuito de aproximar os adolescentes do serviço da APS, é essencial que os profissionais de saúde não realizem apenas atividades pontuais, mas sim que despertem o interesse desses jovens para conhecerem e frequentarem esse espaço. Os profissionais precisam ser os responsáveis pela busca ativa dos adolescentes, de maneira a articular o serviço de saúde com as escolas e os espaços sociais, para que seja viável realizar ações perenes de cuidado (PINTO AC, et al., 2017).

Nesse sentido, ao pensar em estratégias que diminuam o distanciamento entre os adolescentes e os serviços de APS, tem-se a possibilidade de implementar o uso das tecnologias de informação nas unidades de saúde (PINTO LF e ROCHA CMF, 2016). Estas, além de dinamizarem o fluxo de informações, podem contribuir para a produção e divulgação do conhecimento, ampliar canais de comunicação com a população, possibilitar uma aproximação e criação de vínculo entre usuários e profissionais (ATHERTON H, 2003; PINTO LF e ROCHA CMF, 2016).

Nesse contexto, destaca-se que aproximadamente 10 milhões de adolescentes no mundo fazem uso diário da internet, com acessos relacionados, predominantemente, às redes sociais, ao entretenimento e à procura por informações, e dessa forma, faz-se necessário realizar a captação desses adolescentes e capacitar os profissionais de saúde para a utilização desses ambientes virtuais (UNICEF, 2013). As redes sociais mais utilizadas pelos adolescentes são Facebook, Twitter e Instagram, o que possibilita que ações possam ser 
realizadas pelos profissionais nessas plataformas, a fim de alcançar o público jovem e desenvolver ações de educação em saúde voltadas para as necessidades evidenciadas (ARAGÃO JMN, et al., 2018).

A utilização de tecnologias de comunicação/mídias digitais como estratégia de promoção de saúde nos serviços de APS, pode auxiliar no processo de construção da autonomia do adolescente. Isso porque oportunizam um ambiente favorável e acolhedor às diferentes opiniões e formas de expressão, além de estabelecer uma relação horizontal entre os profissionais de saúde e este público (PINTO AC, et al., 2017).

Nesse sentido, este estudo orientou-se pela questão de pesquisa: Quais as evidências científicas relacionadas ao uso da internet como ferramenta de apoio voltada ao público adolescente na atenção primária à saúde? Para respondê-la, objetivou-se: identificar as evidências científicas relacionadas ao uso da internet como ferramenta de apoio voltada ao público adolescente na atenção primária à saúde.

\section{MÉTODOS}

Trata-se de um estudo de revisão integrativa da literatura, técnica que permite a combinação de novos e antigos saberes para a inclusão dos resultados de estudos na prática clínica, de acordo com as evidências científicas. Nessa perspectiva, a revisão permite a qualificação do conhecimento sobre determinada temática, visto que permite uma avaliação crítica e a síntese das evidências disponíveis sobre o tema investigado (SOUSA LLM, et al, 2017; PAULA CC, et al, 2016).

Para a realização do estudo, foram desenvolvidas seis etapas distintas, sendo elas: identificação do tema e seleção da questão de pesquisa; estabelecimento de critérios para inclusão e exclusão de estudos/literatura; definição das informações a serem extraídas dos estudos selecionados; avaliação dos estudos incluídos; interpretação dos resultados e, por último, apresentação e síntese do conhecimento (MENDES KDS, et al, 2008).

Na primeira etapa, estabeleceu-se a definição do problema e a formulação da questão de pesquisa, onde utilizou-se a estratégia PICo, composta por elementos fundamentais quando há pretensão de estudar fenômenos mais específicos. Esta representa um acrônimo para paciente (P), interesse (I) e contexto (Co) (SANTOS CMC, et al, 2007). Assim, definiu-se a seguinte questão de pesquisa: "Quais as evidências científicas relacionadas ao uso da internet como ferramenta de apoio voltada ao público adolescente na atenção primária à saúde?". Nela, os elementos foram: $(P)$ adolescentes, $(I)$ uso da internet como ferramenta de apoio e (Co) Atenção Primária à Saúde.

Na segunda etapa do processo de pesquisa, foram deliberados os critérios de elegibilidade dos estudos. Para isso, incluíram-se artigos originais nos idiomas português, inglês ou espanhol, dos últimos cinco anos, com a intenção de identificar a produção de conhecimento atual, tendo em vista a ampliação e popularização do acesso à internet. Foram excluídos artigos com textos indisponíveis online ou que não respondessem à questão de pesquisa, bem como teses, dissertações e monografias, editoriais e revisão da literatura.

A busca foi realizada durante o mês de julho de 2020 na base de dados eletrônica Medical Literature Analysis and Retrieval System Online (MEDLINE) via Portal PubMed, e nas bases de dados Literatura Latinoamericana e do Caribe em Ciências da Saúde (LILACS) e Base de Dados de Enfermagem (BDEnf) via Biblioteca Virtual em Saúde (BVS). Para tanto, utilizou-se a seguinte estratégia de busca no portal PubMed: [Internet] AND [Teenager] AND [Primary health care]. E no portal BVS, foram utilizados os descritores [Internet] AND [Teenager] AND [Primary health care] OR [Internet] AND [Adolescente] AND [Atenção primária em saúde].

$\mathrm{Na}$ etapa seguinte, deu-se a organização das informações, formando um banco de dados para garantir fácil controle e manejo dos estudos. Desse modo, realizou-se a leitura criteriosa dos títulos, resumos e palavras chave de todas as publicações localizadas pela estratégia de busca, após aplicou-se os critérios de inclusão e exclusão do estudo. Através de uma tabela no programa Microsoft Excel $\AA^{\circledR}$, registrou-se os estudos selecionados e excluídos, por meio de uma ferramenta que possibilitou classifica-los como: não pertencente à temática, não responde à questão de pesquisa e não apresenta o texto completo disponível online. 
A seleção dos estudos foi realizada por dois pesquisadores, simultaneamente. Em situação de divergência, buscou-se um consenso com a participação de um pesquisador auxiliar. A busca teve como resultado 67 artigos, sendo que destes, cinco na base de dados Lilacs, um da BDEnf, e 61 da Medline. Após a leitura de títulos, resumos, palavras-chave e textos na íntegra foram excluídos 58 estudos, dos quais: dois por não pertencerem à temática e 56 por não responderem à questão de pesquisa (Figura 1).

Figura 1 - Fluxograma do processo de inclusão e exclusão dos estudos encontrados.

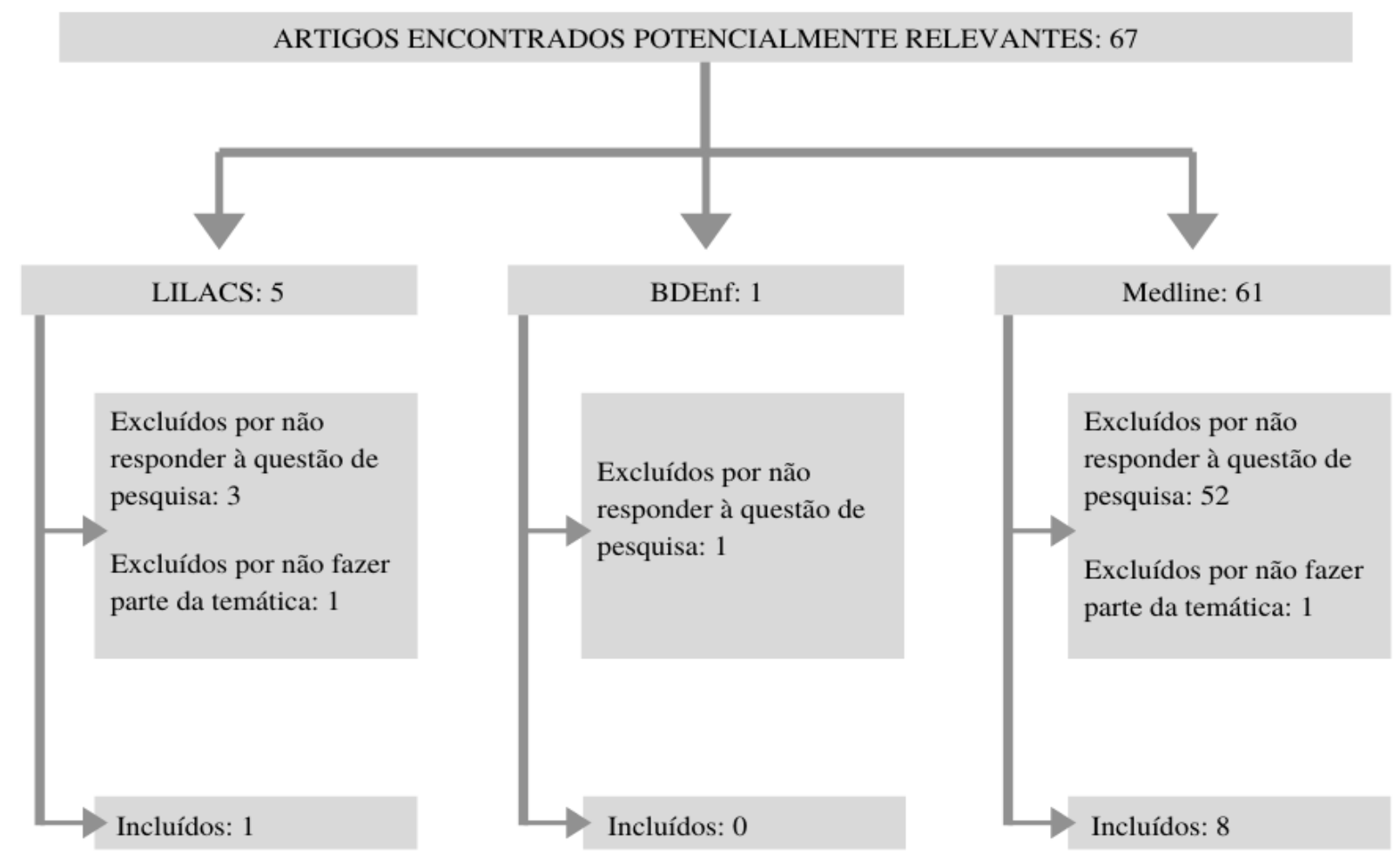

Fonte: SCOPEL MF, et al., 2021.

Durante a quarta etapa, foi realizada a avaliação das informações extraídas dos artigos que foram selecionados na fase anterior. Nesta, a análise foi realizada criteriosamente, buscando informações e explicações para resultados conflitantes entre os estudos. Além disso, classificaram-se os níveis de evidência científica de cada artigo, segundo a proposta dos autores Melnyk BM e Fineout-Overhol E (2011), ou seja, por meio da análise de questão de pesquisa. Sendo assim, as questões clínicas dos estudos primários nortearam a identificação dos níveis, que são organizados em três pirâmides, sendo a primeira delas quando a questão está relacionada a tratamento ou intervenção, a segunda quanto às questões de prognóstico ou etiologia e a terceira pirâmide envolve questões de significado, experiência ou sentimentos.

$\mathrm{Na}$ etapa seguinte, fez-se a interpretação dos principais resultados, por meio da comparação com o conhecimento teórico, identificando conclusões e implicações importantes para a prática. A sexta e última etapa, compreendeu a síntese do conhecimento, incluindo informações suficientes que permitiram a avaliação adequada dos procedimentos realizados no desenvolvimento do estudo, os aspectos relativos ao tópico abordado e o detalhamento dos estudos incluídos, considerando os principais resultados extraídos da análise dos artigos selecionados.

Por fim, no que se refere aos aspectos éticos, foram respeitadas as ideias, as definições e os conceitos empregados pelos autores dos estudos analisados, que foram apresentados e citados de forma fiel e incontestável. 


\section{RESULTADOS E DISCUSSÃO}

O corpus do estudo foi composto por nove artigos, publicados entre os anos de 2015 e 2019. Os artigos foram numerados de A1 à A9. Destes, seis foram realizados nos Estados Unidos da América, enquanto que Alemanha, Inglaterra e Brasil contemplam a revisão com um estudo cada. Em relação ao delineamento metodológico, predominou o Ensaio Clínico Randomizado, abordagem presente em cinco estudos seguido por três estudos qualitativos e um estudo quantitativo (Quadro 1).

Quadro 1 - Síntese dos estudos selecionados, segundo autores e ano, título do trabalho, delineamento metodológico, país de realização e periódico de publicação.

\begin{tabular}{|c|c|c|c|c|}
\hline № & Autores e ano & Título & $\begin{array}{l}\text { Delineamento } \\
\text { metodológico }\end{array}$ & País e periódico \\
\hline $\mathrm{A} 1$ & $\begin{array}{l}\text { BALDOFSKI } \\
\text { S, et al., } \\
2019\end{array}$ & $\begin{array}{l}\text { Efficacy and cost-effectiveness of two } \\
\text { online interventions for children and } \\
\text { adolescents at risk for depression } \\
\text { (E.motion trial): study protocol for a } \\
\text { randomized controlled trial within the } \\
\text { ProHEAD consortium. }\end{array}$ & $\begin{array}{l}\text { Ensaio Clínico } \\
\text { Randomizado }\end{array}$ & $\begin{array}{c}\text { Alemanha; } \\
\text { Springer Nature }\end{array}$ \\
\hline A2 & $\begin{array}{l}\text { PRADO G, et } \\
\text { al., } \\
2019\end{array}$ & $\begin{array}{l}\text { Rationale and design for eHealth } \\
\text { Familias Unidas Primary Care: A drug } \\
\text { use, sexual risk behavior, and STI } \\
\text { preventive intervention for hispanic } \\
\text { youth in pediatric primary care clinics. }\end{array}$ & $\begin{array}{l}\text { Ensaio Clínico } \\
\text { Randomizado }\end{array}$ & $\begin{array}{l}\text { Estados Unidos da } \\
\text { América; } \\
\text { Contemporary } \\
\text { Clinical Trials. }\end{array}$ \\
\hline A3 & $\begin{array}{l}\text { PALERMO } \\
\text { TM, et al., } \\
2018\end{array}$ & $\begin{array}{l}\text { Mobile health intervention for self- } \\
\text { management of adolescent chronic } \\
\text { pain (WebMAP mobile): Protocol for a } \\
\text { hybrid effectiveness-implementation } \\
\text { cluster randomized controlled trial. }\end{array}$ & $\begin{array}{l}\text { Ensaio Clínico } \\
\text { Randomizado }\end{array}$ & $\begin{array}{l}\text { Estados Unidos da } \\
\text { América; } \\
\text { Contemporary } \\
\text { Clinical Trials }\end{array}$ \\
\hline A4 & $\begin{array}{l}\text { BANSA M, et } \\
\text { al., } \\
2018\end{array}$ & $\begin{array}{l}\text { A Little Effort Can Withstand the } \\
\text { Hardship: Fielding an Internet-Based } \\
\text { Intervention to Prevent Depression } \\
\text { among Urban Racial/Ethnic Minority } \\
\text { Adolescents in a Primary Care Setting. }\end{array}$ & $\begin{array}{l}\text { Ensaio Clínico } \\
\text { Randomizado }\end{array}$ & $\begin{array}{l}\text { Estados Unidos da } \\
\text { América; } \\
\text { Journal of the } \\
\text { National Medical } \\
\text { Association }\end{array}$ \\
\hline A5 & $\begin{array}{l}\text { HAUSMANN } \\
\text { JS, et al., } \\
2017\end{array}$ & $\begin{array}{l}\text { Adolescent and Young Adult Use of } \\
\text { Social Media for Health and Its } \\
\text { Implications. }\end{array}$ & Qualitativo & $\begin{array}{l}\text { Estados Unidos da } \\
\text { América; } \\
\text { Journal of Adolescent } \\
\text { Health }\end{array}$ \\
\hline A6 & $\begin{array}{l}\text { MOLLEDA L, } \\
\text { et al., } \\
2017\end{array}$ & $\begin{array}{l}\text { Clinic Personnel, Facilitator, and } \\
\text { Parent Perspectives of eHealth } \\
\text { Familias Unidas in Primary Care. }\end{array}$ & Qualitativo & $\begin{array}{l}\text { Estados Unidos da } \\
\text { América; } \\
\text { Journal of Pediatric } \\
\text { Health Care }\end{array}$ \\
\hline A7 & $\begin{array}{l}\text { PARK MH, et } \\
\text { al., } \\
2015\end{array}$ & $\begin{array}{l}\text { Development and evaluation of an } \\
\text { online tool for management of } \\
\text { overweight children in primary care: a } \\
\text { pilot study. }\end{array}$ & Qualitativo & $\begin{array}{l}\text { Inglaterra; } \\
\text { British Medical } \\
\text { Journal }\end{array}$ \\
\hline A8 & $\begin{array}{l}\text { GLADSTONE } \\
\text { TG, et al., } \\
2015\end{array}$ & $\begin{array}{c}\text { An internet-based adolescent } \\
\text { depression preventive intervention: } \\
\text { study protocol for a randomized control } \\
\text { trial. }\end{array}$ & $\begin{array}{l}\text { Ensaio Clínico } \\
\text { Randomizado }\end{array}$ & $\begin{array}{l}\text { Estados Unidos da } \\
\text { América; } \\
\text { Journal of Clinical } \\
\text { Trials } \\
\end{array}$ \\
\hline A9 & $\begin{array}{l}\text { GONDIN PS, } \\
\text { et al., } \\
2015\end{array}$ & $\begin{array}{l}\text { Acessibilidade dos adolescentes às } \\
\text { fontes de informações sobre saúde } \\
\text { sexual e reprodutiva / Accessibility of } \\
\text { adolescents to sources of information } \\
\text { on sexual and reproductive health }\end{array}$ & Quantitativo & $\begin{array}{c}\text { Brasil; } \\
\text { Revista Brasileira de } \\
\text { Crescimento e } \\
\text { Desenvolvimento } \\
\text { Humano }\end{array}$ \\
\hline
\end{tabular}

Fonte: SCOPEL MF, et al., 2021. 
A análise e síntese dos dados da revisão integrativa possibilitou o agrupamento dos estudos em três categorias temáticas: "Intervenções online que visam a prevenção de agravos e promoção da saúde dos adolescentes", "Ferramentas adotadas pelo serviço para assistência à saúde mental dos adolescentes" e "Uso da internet pelos adolescentes como fonte de informação em saúde".

\section{Intervenções online que visam a prevenção de agravos e promoção da saúde dos adolescentes}

De acordo com os estudos A2, A3, A6 e A7, identificaram-se algumas barreiras quanto à implementação efetiva de intervenções online na APS. Uma das questões diz respeito à precariedade de recursos tecnológicos essenciais para a implementação desses serviços. Outra necessidade foi a aceitabilidade das tecnologias para o cuidado a adolescentes por parte dos profissionais, devido às restrições de tempo e espaço físico nas unidades para que as equipes façam as adequações necessárias, além da problemática de longas filas de espera (Quadro 2).

Quadro 2 - Caracterização dos estudos incluídos na primeira categoria segundo título, nível de evidência científica e objetivos.

\begin{tabular}{|c|c|c|}
\hline No & Nível de evidência & Objetivos \\
\hline A2 & II & $\begin{array}{r}\text { Determinar o custo e a eficácia do programa online "eHealth Familias } \\
\text { Unidas Primary Care" na atenção primária a partir da integração de } \\
\text { intervenções em saúde dos adolescentes, para identificar a efetividade } \\
\text { na redução do uso de drogas, sexo desprotegido e incidência de IST, } \\
\text { com apoio da melhoria do funcionamento familiar. }\end{array}$ \\
\hline A3 & II & $\begin{array}{r}\text { Determinar a eficácia de um aplicativo online para melhorar resultados } \\
\text { na saúde do adolescente com dor crônica, e identificar os impactos das } \\
\text { intervenções realizadas pela atenção primária. }\end{array}$ \\
\hline A6 & II & $\begin{array}{r}\text { Compreender a viabilidade e a aceitabilidade da implementação de } \\
\text { plataforma por meio de uma intervenção online, familiar e para } \\
\text { adolescentes na atenção primária. }\end{array}$ \\
\hline A7 & VI & $\begin{array}{r}\text { Explorar a aceitabilidade da implementação de uma ferramenta on-line } \\
\text { para avaliação e gestão da obesidade infantil (Tratamento Assistido por } \\
\text { Computador de CHildren, CATCH) na atenção primária. }\end{array}$ \\
\hline
\end{tabular}

Fonte: SCOPEL MF, et al., 2021.

O acesso a um dispositivo conectado à rede mostra-se como alternativa de intervenção de saúde ao público adolescente, servindo como meio de divulgação de ações, programas, campanhas, estratégias e realização de consultas, individuais ou coletivas, sem a necessidade de deslocamento a uma unidade física. Com isso, reforça-se práticas educativas para promoção de saúde e, consequente, autonomia para a mudança de hábitos para uma vida saudável (DOS SANTOS CA, et al, 2019).

Os achados a respeito dos benefícios possíveis advindos da utilização de tecnologias para intervenções em saúde nos adolescentes são promissores. Segundo o estudo $A 3$, os aplicativos que podem ser utilizados por meio de smartphones, sites e mensagens de texto são um meio ideal e de fácil acesso para fornecer o suporte necessário. É possível inferir, segundo os trabalhos A6 e A7, que o fato de os adolescentes estarem em suas casas ou ambientes confortáveis, torna a experiência mais agradável, pois apresentaram-se mais dispostos e interessados em buscar informações e compartilhar vivências com os profissionais de saúde.

Destaca-se, o processo de adaptação dos profissionais de saúde para o avanço das tecnologias e a familiarização dos jovens com essa ferramenta. Assim, com o intento de contemplar as demandas apresentadas pelos usuários, surge a necessidade de capacitar os profissionais responsáveis pelas orientações, por meio de Educação Permanente em Saúde (EPS) para a resolução de adversidades e melhorar $\mathrm{o}$ atendimento remoto conforme a condição de saúde do adolescente (MOLLEDA L, et al, 2017; PRADO $G$, et al, 2019). A EPS é a fusão dos conhecimentos adquiridos na teoria acadêmica com as singularidades exigidas na prática, a fim de manter a assistência em concordância com a realidade de sua comunidade adstrita (BRASIL, 2018). 
Sobretudo, há uma preocupação expressiva, aparente nos estudos supracitados, no que compete à demanda, pois em função do dimensionamento de pessoal e do fluxo de funcionamento de uma unidade de saúde, o tempo destinado ao atendimento pela internet será exíguo. Como efeito, podem aparecer dificuldades em continuar os encontros regularmente e resultar em baixa adesão ao acompanhamento. Isso pode refletir em uma redução na qualidade do atendimento virtual, revelando-se como importantes fatores limitantes da efetividade da ferramenta (PRADO G, et al, 2019; PALERMO TM, et al, 2018; HAUSMANN JS, et al, 2017).

\section{Ferramentas adotadas pelo serviço voltadas para a saúde mental dos adolescentes}

As intervenções na internet destacaram-se por diferentes motivos: acesso rápido à informação relacionada à saúde e comunidades de apoio aos adolescentes; possibilidade de ofertar ferramentas de autoajuda para os jovens; e por consagrar-se como melhor estratégia para aqueles que não se sentem à vontade ao buscar ajuda presencialmente nas unidades de APS (Quadro 3).

Quadro 3 - Caracterização dos estudos incluídos na segunda categoria, segundo título, nível de evidência científica e objetivos.

\begin{tabular}{|c|c|c|}
\hline No & Nível de evidência & Objetivos \\
\hline A1 & II & $\begin{array}{c}\text { Proporcionar uma melhor compreensão do comportamento de } \\
\text { busca de ajuda de crianças e adolescentes, potenciais } \\
\text { benefícios das intervenções em saúde mental para essa faixa } \\
\text { etária e novas percepções sobre aspectos até agora pouco } \\
\text { estudados em programas de saúde mental. }\end{array}$ \\
\hline A4 & II & $\begin{array}{c}\text { Avaliar o aplicativo desenvolvido para ensinar adolescentes a } \\
\text { lidar com as diferentes situações que podem acarretar danos } \\
\text { emocionais. }\end{array}$ \\
\hline A8 & II & $\begin{array}{c}\text { Avaliar uma abordagem autoguiada e online para a prevenção } \\
\text { da depressão e uma intervenção geral em educação em } \\
\text { saúde na Internet para adolescentes. }\end{array}$ \\
\hline
\end{tabular}

Fonte: SCOPEL MF, et al., 2021.

O estudo A8 destaca que, apesar de os adolescentes apresentarem uso extensivo da internet, há poucos relatos de que eles utilizam as redes de contato online na busca de ajuda quando se sentem prejudicados com determinadas situações em sua vida. O estudo conclui que programas baseados na Internet são mais eficazes quando estão relacionados a estratégias de engajamento, tanto dentro do site de intervenção quanto na vida real. Assim, programas eficazes de prevenção à depressão na Internet incorporam esses princípios em seu desenho e oferecem estratégias adicionais para promover oportunidades de identificação, para engajar o processo de mudança de pensamento e comportamento. Ainda, revelou-se uma lacuna investigativa no que diz respeito às ferramentas de amparo à saúde dos adolescentes, visto que, em caráter brasileiro, há poucas análises que aproximam essa população ao serviço de APS e inexistem ferramentas específicas que favoreçam essa proximidade (MOLLEDA L, et al, 2017).

Ressalta-se que a depressão é um fator de risco importante para a saúde do adolescente, em especial quando a situação se agrava sem tratamento e acompanhamento adequado. As intervenções pela internet apresentaram uma abordagem promissora para superar as barreiras no atendimento (DOGAN E, et al, 2017). O uso das mídias sociais é visualizado como uma ferramenta propulsora de educação em saúde para o cuidado de adolescentes, contudo o relacionamento da equipe multiprofissional precisa ser integrado, levando em conta as possibilidades de olhar singular para cada área específica. Somente após a consolidação do bom relacionamento do grupo profissional, será possível visualizar meios para a conexão entre o serviço de saúde e a população alvo (MOLLEDA L, et al, 2017).

Por meio do estabelecimento de vínculo entre a equipe de saúde e os adolescentes, o relacionamento virtual precisa fluir de forma natural e não hierárquica. A comodidade do adolescente estar em sua casa 
contribui para a construção e conservação do elo e, consequentemente, facilita a exteriorização de questões anteriormente consideradas vergonhosas, não apenas em encontros, como também em mídias meramente informativas disponibilizadas pelo serviço de saúde (MOLLEDA L, et al, 2017; PARK MH, et al, 2015).

Ademais, a operacionalização do sistema ofertado precisa ser atraente e despertar o interesse dos adolescentes, instigando suas curiosidades e fomentando suas dúvidas. Com o intuito de que os usuários permaneçam fazendo o uso do instrumento, sugere-se que os programas interajam, por meio de notificações, e forneçam informações de acesso rápido e fácil compreensão (GLADSTONE TG, et al, 2015). A partir da possibilidade do uso de tecnologias, as barreiras que distanciavam os adolescentes do serviço de saúde são diminuídas e é viabilizado o acesso à assistência à saúde com qualidade, resultando em práticas de prevenção e promoção da saúde.

\section{Uso da internet pelos adolescentes como fonte de informação em saúde}

Diante da resistência em procurar os serviços presenciais de saúde e utilização de grande parte do seu tempo diário em espaços virtuais, uma estratégia para aproximar este público refere-se ao uso da Internet como um meio para viabilizar a busca de informações corretas e de qualidade a respeito da sua saúde (Quadro 4).

Quadro 4 - Caracterização dos estudos incluídos na terceira categoria segundo título, nível de evidência científica e objetivos.

\begin{tabular}{|c|c|c|}
\hline № & Nível de evidência & Objetivos \\
\hline A5 & IV & $\begin{array}{r}\text { Estudo para determinar como adolescentes usam as mídias sociais } \\
\text { para compartilhar informações de saúde e avaliar atitudes em relação } \\
\text { ao uso das mídias sociais para obter informações de saúde e se } \\
\text { comunicar com os provedores médicos. }\end{array}$ \\
\hline A9 & IV & $\begin{array}{r}\text { Identificar as fontes de informações utilizadas pelos adolescentes da } \\
\text { escola pública sobre sexualidade e reprodução }\end{array}$ \\
\hline
\end{tabular}

Fonte: SCOPEL MF, et al., 2021.

Os estudos apresentados no Quadro 4 (A5, A9) ratificam as informações sobre dificuldades enfrentadas na implantação de plataformas online e de redes sociais com adolescentes para informação em saúde. Embora o espaço online esteja sendo cada vez mais utilizado para sanar dúvidas a respeito dos processos de saúde-doença, ainda é considerado principalmente como um local de recreação e socialização. Isso é evidenciado de forma a enfatizar que a procura indiscriminada de informações, especialmente voltada à sexualidade, e sem o auxílio necessário pode resultar em captação de dados, muitas vezes, falsos e de procedência duvidosa. Em vista disso, podem desenvolver práticas equivocadas e prejudiciais a sua integridade física e psicossocial (GONDIM PS, et al, 2015).

De acordo com o estudo A9, existe uma forte influência da família e da escola no que diz respeito ao comportamento sexual dos jovens e seus consequentes riscos e problemas de saúde. Torna-se fundamental, portanto, que sejam disponibilizadas informações envolvendo os adolescentes e suas redes de apoio, incluindo amigos, família e escola em discussões e divulgando periodicamente projetos de educação em saúde em ambientes online acessados pelos jovens, bem como nos ambientes escolares e sociais.

O fato de que a maior procura na internet pelos jovens é para recreação e em poucos casos na resolução de dúvidas que remetem a situações de saúde/doença, principalmente, sobre assuntos ligados à sexualidade é um fato preocupante comum aos artigos dessa categoria. Essa procura de forma indiscriminada e sem o auxílio necessário pode resultar em captação de dados muitas vezes falsos, sem evidências e de procedência duvidosa. Em vista disso, podem desenvolver práticas equivocadas e prejudiciais a sua integridade física e psicossocial (GONDIM PS, et al, 2015). As ferramentas virtuais são mais eficazes quando utilizadas adjuntas de estratégias de engajamento, podendo ser delineadas por meio de discussões que englobem a opinião dos profissionais de saúde juntamente com os adolescentes, famílias e escola. Isto pode facilitar o conhecimento 
da realidade dos adolescentes, a realização de ações conjuntas de promoção à saúde e a disseminação de informações seguras de fácil acesso e entendimento.

A utilização da internet, portanto, requer que os adolescentes e familiares estejam conscientes dos riscos aos quais são expostos, além de maneiras de evitar problemas quanto à segurança digital. O uso seguro da Internet requer habilidades na utilização dos meios digitais a fim de proteger dados e saber onde encontrar ferramentas disponíveis para proteção. Assim, deve-se considerar que o ideal é manter cautela na internet quanto ao fornecimento de informações, entendendo-a como um local público onde qualquer pessoa poderá ter acesso ao que é divulgado (BRASIL, 2014).

\section{CONSIDERAÇÕES FINAIS}

As evidências científicas relacionadas ao uso da internet como ferramenta de apoio voltada ao público adolescente na atenção primária à saúde, indicaram uma melhora na adesão dos adolescentes à procura dos serviços de saúde bem como uma possibilidade de fornecer informação de qualidade a esse grupo. Evidenciou-se, ainda, que a facilidade e a praticidade de poderem acessar, de suas residências, viabiliza uma condição de maior conforto para dialogar com o profissional de saúde, minimizando episódios de constrangimento e omissão de informações, especialmente, acerca de temas da esfera íntima e privada. Como fatores limitantes, aponta-se a precariedade de recursos, baixa aceitabilidade das tecnologias por parte dos profissionais de saúde devido às restrições de tempo e espaço físico.

\section{AGRADECIMENTOS E FINANCIAMENTO}

Agradecemos ao Programa Fundo de incentivo de extensão (FIEX), ao Programa CNPq - Chamada Universal e ao Programa FIPE SÊNIOR da UFSM pelas bolsas de Iniciação Científica.

\section{REFERÊNCIAS}

1. ARAGÃO JMN, et al. The use of Facebook in health education: perceptions of adolescent students. Revista Brasileira de Enfermagem, 2018; 71 (2): 265-71.

2. ATHERTON H. Use of email for consulting with patients in general practice. British Journal of General Practice, 2013; 63 (608): 11811-11819.

3. BALDOFSKI S, et al. Efficacy and cost-effectiveness of two online interventions for children and adolescents at risk for depression (E. motion trial): study protocol for a randomized controlled trial within the ProHEAD consortium. Trials, 2019; 20 (1): 53.

4. BANSA M, et al. A little effort can withstand the hardship: fielding an internet-based intervention to prevent depression among urban racial/ethnic minority adolescents in a primary care setting. Journal of the National Medical Association, 2018; 110 (2): 130-142.

5. Básica. 1 ed. Departamento de Ações Programáticas e Estratégicas. Brasília: Ministério da Saúde, 234 p. 2017.

6. BRASIL. Ministério da Justiça. Classificação indicativa e novas mídias. 1 ed. Brasília: Ministério da Justiça. Secretaria Nacional de Justiça, 2014; 3: 182.

7. BRASIL. Ministério da Saúde. Política Nacional de Educação Permanente em Saúde: o que se tem produzido para o seu fortalecimento? 1 ed. Secretaria de Gestão do Trabalho e da Educação na Saúde. Departamento de Gestão da Educação na Saúde. Brasília: Ministério da Saúde, 2018; 78p.

8. BRASIL. Ministério da Saúde. Proteger e Cuidar da Saúde de Adolescentes na Atenção. 2017.

9. COSTA RF, et al. Redes de apoio ao adolescente no contexto do cuidado à saúde: interface entre saúde, família e educação. Revista da Escola de Enfermagem da USP, 2015; 49 (5): 741-747.

10. DOGAN E, et al. Smartphone-based monitoring of objective and subjective data in affective disorders: where are we and where are we going? Systematic review. Journal of medical Internet research, 2017; 19 (7): e262.

11. DOS SANTOS CA, et al. Internet e HPV: Uma possibilidade para educação em saúde entre adolescentes?. Adolescência e Saúde, 2019; 16 (1): 46-59.

12. GLADSTONE TG, et al. An internet-based adolescent depression preventive intervention: study protocol for a randomized control trial. Trials, 2015; $16(1): 1-17$.

13. GONDIM PS, et al. Acessibilidade dos adolescentes às fontes de informações sobre saúde sexual e reprodutiva. Revista Brasileira de Crescimento e Desenvolvimento Humano, 2015; 25 (1). 
14. HAUSMANN JS, et al. Adolescent and young adult use of social media for health and its implications. Journal of Adolescent Health, 2017; 60 (6): 714-719.

15. MELNYK BM, FINEOUT-OVERHOLT E. Making the case for evidence-based practice and cultivating a spirit of inquiry. Evidence-based practice in nursing \& healthcare: A guide to best practice. Philadelphia: Wolters Kluwer, Lippincott Williams \& Wilkins. p.3-24. 2011.

16. MENDES KDS, et al. Revisão integrativa: método de pesquisa para incorporação de evidências na saúde e na enfermagem. Texto contexto - Enfermagem, 2008; 17 (4): 758-764.

17. MOLLEDA L, et al. Clinic personnel, facilitator, and parent perspectives of eHealth Familias Unidas in primary care. Journal of Pediatric Health Care, 2017; 31 (3): 350-361.

18. PALERMO TM, et al. Mobile health intervention for self-management of adolescent chronic pain (WebMAP mobile): Protocol for a hybrid effectiveness-implementation cluster randomized controlled trial. Contemporary clinical trials, 2018; 74: 55-60.

19. PARK MH, et al. Development and evaluation of an online tool for management of overweight children in primary care: a pilot study. BMJ open, 2015; 5 (6).

20. PAULA CC, et al. Revisão integrativa como ferramenta para tomada de decisão na prática em saúde. Metodologias da pesquisa para a enfermagem e saúde da teoria à prática. Porto Alegre: Moriá, p. 51-76. 2016.

21. PESSOA DMS, et al. Assistência de Enfermagem na Atenção Primária à Saúde de adolescentes com ideações suicidas. Revista Mineira de Enfermagem, 2020; 24: e-1290.

22. PINTO AC, et al. Uso de tecnologias da informação e comunicação na educação em saúde de adolescentes: revisão integrativa. Revista de Enfermagem UFPE online, 2017; 11 (2): 634-644.

23. PINTO LF, ROCHA CMF. Inovações na Atenção Primária em Saúde: o uso de Ferramentas de tecnologia de comunicação e informação para apoio à gestão local. Revista Ciência \& Saúde Coletiva, 2016; 21 (5): 1433-1448.

24. PRADO G, et al. Rationale and design for eHealth Familias Unidas Primary Care: A drug use, sexual risk behavior, and STI preventive intervention for hispanic youth in pediatric primary care clinics. Contemporary clinical trials, 2019; 76: 64-71.

25. SANTOS CMC, et al. The PICO strategy for the research question construction and evidence search. Revista LatinoAmericana de Enfermagem, 2007; 15 (3): 508-511.

26. SOUSA LLM, et al. Metodologia de Revisão Integrativa da Literatura em Enfermagem. Revista Investigação Enfermagem, 2017; 2: 17-26.

27. UNICEF. Fundo das nações unidas para a infância. O uso da internet por adolescentes. Brasília, DF: UNICEF. 2013. 